\title{
RETROPERITONEAL HYDATD CYST: A RARE PRESENTATION
}

Dr. Siddesh. Basavaraj. Sirwar, Dr. Amrutha. Swati. Indupalli, Dr. Subhanulla P.

1. Professor, Department of Microbiology. Khajabanda Nawaz Institute of Medical Sciences, Gulbarga.

2. Associate Professor, Department of Community Medicine, Khajabanda Nawaz Institute of Medical Sciences.

3. Professor, Department of Surgery, Khajabanda Nawaz Institute of Medical Sciences, Gulbarga.

\section{CORRESPONDING AUTHOR:}

Dr. Siddesh. Basavaraj. Sirwar,

Mathru Nilaya, Plot No: 11, Siddeshwara Colony,

Old Jewargi Road, Gulbarga -02, Karnataka,

Email id- drsidd1110@gmail.com,

Ph- 009109448320166 / 09902536576.

ABSTRACT: Primary retro peritoneal hydatid cyst is extremely rare and only occasional case reports have appeared since first reported this entity in 1958. We report this rare case from KBN Hospital Gulbarga Karnataka diagnosis confirmed post operatively. Mass approached through greater sac which revealed a single cavity in retroperitonium containing enormous number of typical cyst of $2-5 \mathrm{cms}$ diameter occupying sub hepatic, epigastria, umbilical and left lumbar regions extending to pelvis. It was never expected that such enormous 2970 cysts would be recovered. Hence, suspicion and ruling out hydatid cyst in every case of retroperitoneal cystic swelling especially in endemic areas, and a careful search for hydatid cysts in other uncommon sites should be cogitated.

KEY WORDS: Hydatid Cyst, Retro Peritoneum.

INTRODUCTION: Hydatid disease is a parasitic infestation by a tapeworm of the genus Echinococcus. Hydatid cysts are a manifestation of infection at the larval stage of Echinococcus granulosus. Human echinococcosis is a zoonotic infection transmitted by dogs in livestock raising areas.

Developing countries with poor hygiene, where sheep and cattle are raised are high-risk areas of acquiring this disease. Hydatid disease (Echinococcus granulosus) is endemic in the Middle East as well as other parts of the world, Africa, South America, New Zealand, Australia, Turkey and Southern Europe including India ${ }^{1-3}$, Foci of hydatid disease also exists in India where the highest prevalence is reported in Andhra Pradesh and Tamil Nadu than in other parts of the country4.

Echinococcus granulosus causes cystic echinococcosis in humans. This relatively benign parasitic disease is characterized by slowly growing cysts most commonly in the liver(accounting for $50-70 \%$ of cases), followed by the lungs(20-30\%), and less frequently the spleen, kidneys, heart, bones, central nervous system, and other organs 5 .

The annual incidence of hydatid disease has been reported to be 18 to 20 cases per 100.000 inhabitants $^{6}$. Retroperitoneal localization of hydatid cyst is unusual ${ }^{7}$ here we are reporting a case of primary retroperitoneal hydatid cyst with unusual number which is a very rare presentation.

CASE REPORT: A female aged 35 year presented with history of abdominal discomfort and distension of 1 year duration, loss of appetite, fever on and off for 3 months. On examination patient looked pallor. There was no jaundice, no edema, vital signs were, pulse 76/min, blood 
pressure was $110 / 70 \mathrm{mmHg}$ and mild fever $\left(9^{\circ} \mathrm{F}\right)$. Chest auscultation revealed normal breath sounds and heart sounds. On Local Examination of abdomen there was diffuse distension confined to upper abdomen, left flank and lower pelvis. Very soft, non tender mass felt more like fluid but no shifting dullness. Borders were indistinct.

INVESTIGATIONS: Hemoglobin-9.5gm\%, Hypochromic, normocytic anemia, blood cell count $11.500 / \mathrm{mm} 3$, Eosinophil count $550 / \mathrm{mm}^{3}$, erythrocyte sedimentation rate $35 \mathrm{~mm} / \mathrm{h}$. The chest and abdominal ultra sound showed normal liver, spleen, kidneys and multiple cystic lesions in the peritoneal cavity occupying upper abdomen, left flank and parts of pelvis.

Explorative laparotomy was done through upper midline incision Fig -1 .Liver, spleen, kidneys and peritoneum showed no cysts. Lesion appeared to be more posterior. Mass approached through greater sac which revealed a single cavity in retroperitonium containing enormous number of typical cyst of $2-5 \mathrm{cms}$ diameter occupying sub hepatic, epigastria, umbilical and left lumbar regions extending to pelvis . A total of 2970 cysts were delivered Fig- 2 . Examination of contents of cysts in laboratory revealed numerous hooklets, scolices, many pus cells. Histopathological examination also confirmed the diagnosis. ELISA test was positive for both Ig.M, and Ig.G Echinococcus.

DISCUSSION: Hydatid disease in human is one of the oldest diseases known to mankind. Hydatid cysts in humans and animals have been known since Roman times, but, as was true for the other tapeworms, the relationship between the larval cyst and the adult worm was not suspected until the eighteenth century. E. granulosus was described as a separate species in 1850, and its life cycle was worked out with feeding experiments in $1863^{8}$. Echinococcus granulosus, which commonly has a sheep-dog cycle, but which may also infect goats, cattle, swine, and camels, is the most likely to infect human beings. Human echinococcosis occurs primarily in sheep rearing areas. Dogs ingest cysts in the offal of dead sheep and pass eggs in their feces. Humans acquire the eggs from a dog's fur or from contaminated food or water. The adult worm lives in the proximal small bowel of the definitive host, attached by hooklets to the mucosa. Eggs are released into the host's intestine and excreted in the feces. Humans may become intermediate hosts through contact with a definitive host (usually a domesticated dog) or ingestion of contaminated water or vegetables. The ovum loses its protective layer as it is digested in the duodenum. Once the parasitic embryo passes through the intestinal wall to reach the portal venous system or lymphatic system, the liver acts as the first line of defense and is therefore the most frequently involved organ. Secondary involvement due to hematogenous dissemination may be seen in almost any anatomic location ${ }^{9}$. Cysts holding 2 or more liters of fluid and larvae can grow for years in the liver, lungs, brain, or other organs and exert enough mechanical pressure to cause grave or fatal consequences ${ }^{8}$. The vast majority of abdominal and pelvic cysts are considered to be secondary to prior hepatic involvement following spontaneous rupture or surgical inoculation. Isolated primary retroperitoneal location is exceptional. Haemotagenous or lymphatic spread could account for a solitary retroperitoneal lesion ${ }^{10,11}$. Dow $^{12}$ waddle $^{13}$ had favored air borne transmission and direct implantation of the embryo in the bronchial mucosa, which is another possible mode of entry. This raises the possibility of an embryo of the parasite entering a venule after penetrating the bronchial mucosa and reaching the left side of the heart to involve other sites and thus bypassing the lung. But this remains largely theoretical and needs to be proved ${ }^{14}$. Preoperative diagnosis is difficult to make unless circumlinear calcification is seen in the plain x-ray of the abdomen. The diagnosis was missed 
clinically as it was provisionally diagnosed retroperitoneal or peritoneal mass. However after ultra sound examination and during surgery, the possibility of hydatid cyst was considered but it was never expected that such enormous cysts would be recovered. Necessary precautions were taken during the excision and marsupialisation of the entire huge cyst cavity and washed with scolicidal (hypertonic saline) solutions. The rupture will result in anaphylactic shock or systemic metastasis. So the treatment of choice is surgical and complete removal of the cyst is the gold standard but its feasibility is related to the location of the cyst. Hence, one must have a high index of suspicion and rule out hydatid cyst in every case of retroperitonel cystic swelling especially in endemic areas. A careful search for hydatid cysts in other organs of abdomen including liver should be made because secondary retroperitoneal hydatids may out number primary retroperitoneal Hydatids.

\section{FIGURES}

Figure1: showing the surgical approach by Explorative laprotomy through upper midline incision to the cyst.

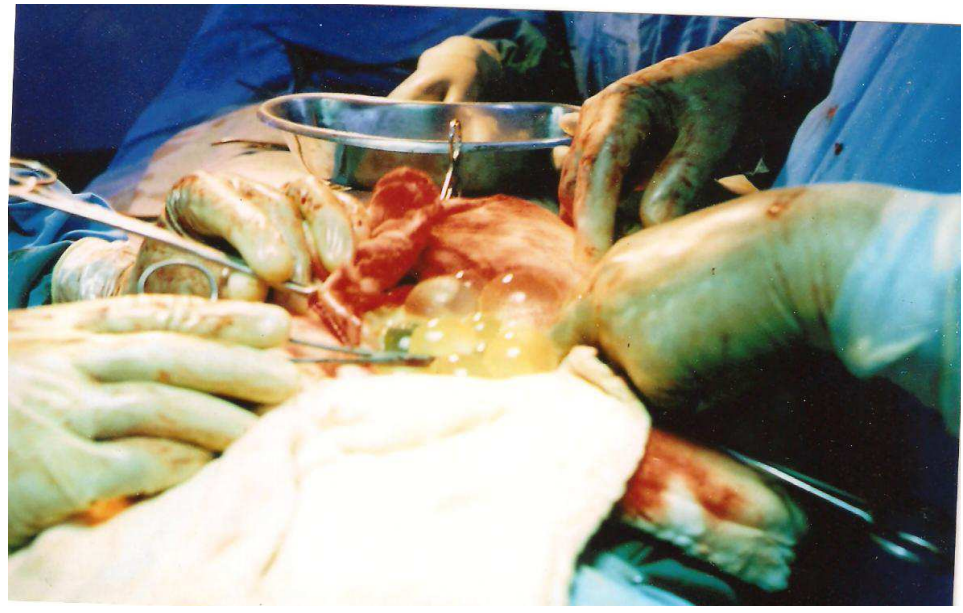

Figure2: Showing unusual number of isolated retro peritoneum Hydatid Cyst.

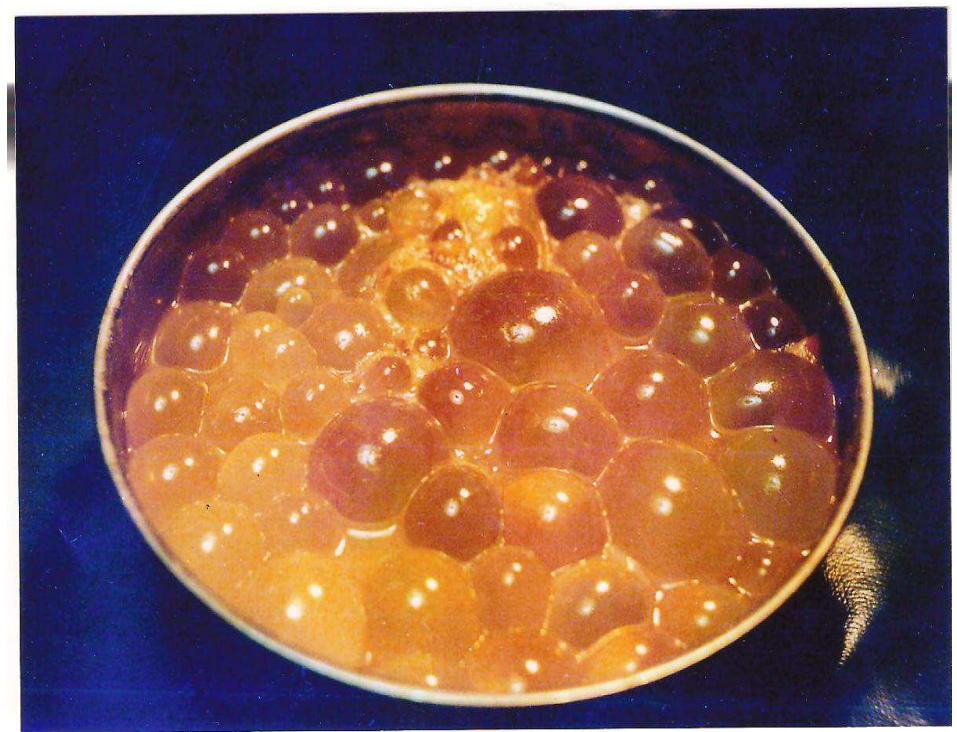




\section{REFERENCES:}

1. Goel MC, Agarwal MR, Misra A.: Percutaneous drainage of renal hydatid cyst: early results and follow-up.:Br J Urol: 1995; 75: 724-8.

2. Altinors N, Senveli E, Donmez T, Bavbek M, Kars Z, Sanli M.; Management of problematic intracranial hydatid cysts;. Infection 1995; 23: 283-7.

3. Brown RA, Millar AIW, Steiner Z, Krige JEJ, Burkimsher D, Cywes S.; Hydarid cyst of the pancreas: a case report in a child. Eur J Pediatr Surg; 1995; 5:121-4.

4. Reddy CRRM. Epidemiology of hydatid disease in Kurnool.Ind; J Med Res; 1968; 56:1205-20.

5. Kammerer WS, Schantz PM.; Echinococcal disease. Infect DisClin North Am 1993; 7:605-18.

6. Özdemir N., Akal M., Kutlay H., Yavuzer Ș. Chest Wall Echinoccocosis. Chest, 1994, 105 : 1277-9.

7. Hatipog Lu A. R, Coșkun Í, Karakaya K, Íbiș C. Retroperitoneal Localization of Hydatid Cyst Disease. Hepato-Gastroenterology, 2001, $48: 1037-9$

8. Patterson, K David, “ Echnicoccosis (Hydatidosis)” The Cambridge World History of Human Disease. Ed. Kenneth F.Kiple. Cambridge University Press 1993. Cambridge Histories Online. Cambridge University Press 11 September 2012 DOI: 10.1017/CHOL9780521332866.105.

9. Pedrosa I, Saiz A, Arrazola L, Ferreiros J, Pedrosa CS. "Hydatid disease: radiologic and pathologic features and complications." RadioGraphics 2000;20:795-817

10. Elton C., Lewis M., Jourdan M. H. Unusual site of hydatid disease. Lancet, 1999, 355 : 2132.

11. Balık I. A. A.,Celebi F., Bașog LU M, Oren,D, Yıldırgan.I, Atmanalp.S.S.: Intra-abdominal extrahepatic echinococcosis. Surg Today, 2001,31:881-4.

12. Dow HR Hydatid disease it's pathology, diagnosis and treatment 1st edition, 1928, 304-315

13. Waddle.N; pulmonary hydatid disease. Aust.N.Z.J Surg, 1950,19:273-78.

14. SekarNN, Madhavan KK, Yadav RV, Katariya RN: Primary retroperitoneal hydatid cyst (A report of three cases \& review of the literature): J. Post grad Med.1982: 28:112-48. 